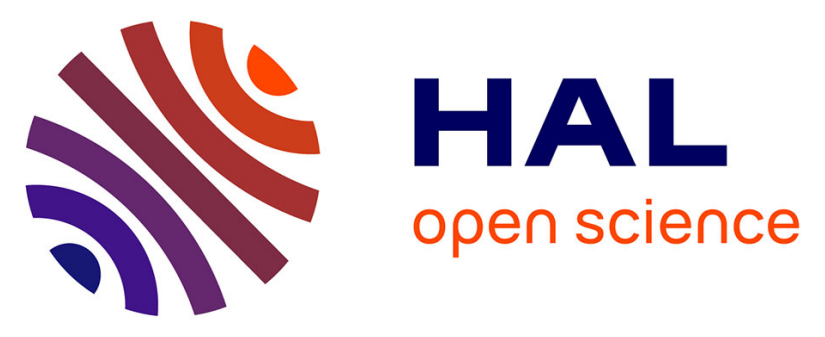

\title{
Prediction of Post-Ablation Outcome in Atrial Fibrillation Using Shape Parameterization and Partial Least Squares Regression
}

Shuman Jia, Claudia Camaioni, Marc-Michel Rohé, Pierre Jaïs, Xavier

Pennec, Hubert Cochet, Maxime Sermesant

\section{To cite this version:}

Shuman Jia, Claudia Camaioni, Marc-Michel Rohé, Pierre Jaïs, Xavier Pennec, et al.. Prediction of Post-Ablation Outcome in Atrial Fibrillation Using Shape Parameterization and Partial Least Squares Regression. FIMH 2017 - International Conference on Functional Imaging and Modeling of the Heart, Jun 2017, Toronto, Canada. pp.314 - 321, 10.1007/978-3-319-59448-4_30 . hal-01574831

\section{HAL Id: hal-01574831 \\ https://hal.inria.fr/hal-01574831}

Submitted on 16 Aug 2017

HAL is a multi-disciplinary open access archive for the deposit and dissemination of scientific research documents, whether they are published or not. The documents may come from teaching and research institutions in France or abroad, or from public or private research centers.
L'archive ouverte pluridisciplinaire HAL, est destinée au dépôt et à la diffusion de documents scientifiques de niveau recherche, publiés ou non, émanant des établissements d'enseignement et de recherche français ou étrangers, des laboratoires publics ou privés. 


\title{
Prediction of Post-Ablation Outcome in Atrial Fibrillation Using Shape Parameterization and Partial Least Squares Regression
}

\author{
Shuman Jia ${ }^{1 \star}$, Claudia Camaioni ${ }^{2}$, Marc-Michel Rohé ${ }^{1}$, Pierre Jaïs ${ }^{2}$, Xavier \\ Pennec $^{1}$, Hubert Cochet $^{2}$, and Maxime Sermesant ${ }^{1}$ \\ 1 Université Côte d'Azur, Asclepios Research Group, Inria, Sophia Antipolis, France \\ 2 IHU Liryc, University of Bordeaux, Pessac, France
}

\begin{abstract}
To analyze left atrial remodeling may reveal shape features related to post-ablation outcome in atrial fibrillation, which helps in identifying suitable candidates before ablation. In this article, we propose an application of diffeomorphometry and partial least squares regression to address this problem. We computed a template of left atrial shape in control group and then encoded the shapes in atrial fibrillation with a large set of parameters representing their diffeomorphic deformation. We applied a two-step partial least squares regression. The first step eliminates the influence of atrial volume in shape parameters. The second step links deformations directly to post-ablation recurrence and derives a few principle modes of deformation, which are unrelated to volume change but are involved in post-ablation recurrence. These modes contain information on ablation success due to shape differences, resulting from remodeling or influencing ablation procedure. Some details are consistent with the most complex area of ablation in clinical practice. Finally, we compared our method against the left atrial volume index by quantifying the risk of post-ablation recurrence within six months. Our results show that we get better prediction capabilities (area under receiver operating characteristic curves $A U C=0.73)$ than left atrial dilation $(A U C=$ 0.47 ), which outperforms the current state of the art.
\end{abstract}

Keywords: atrial fibrillation, catheter ablation, post-ablation outcome, left atrial remodeling, statistical shape analysis, partial least squares regression.

\section{Introduction}

Atrial fibrillation (AF) is the most common type of cardiac arrhythmia [1], characterized by uncoordinated electrical activation and disorganized contraction of the atria. This condition is associated with life-threatening consequences, such as stroke and heart failure. Catheter ablation is an effective treatment for $\mathrm{AF}$ and may be recommended for drug refractory patients. Yet, for $30 \%$ of patients, AF redevelops, resulting in repeated interventions and higher risk.

\footnotetext{
* Email: shuman.jia@inria.fr
} 
In order to optimize the planning of AF treatment, a number of studies have been looking into predictors of recurrent arrhythmia. Previous studies reported that hypertension, Holter duration, left atrial (LA) volume and LA sphericity $[2,3,4,5]$ were potentially related to post-ablation recurrence, but their underlying mechanism is still unclear, as is LA remodeling in AF.

In this exploratory work, we focus on shape variations as predictors. By studying principal deformation modes associated with a higher post-ablation recurrence rate, we expect to find cartographic/regional criteria that will help us stratify the risk of post-ablation recurrence. So far, LA volume index (LAVI) has been the only clinically accessible and reproductive index for identifying suitable candidates for ablation. However, its relation to post-ablation outcome has been constantly reported to be weak [6]. We therefore decided to explore shape features that are more comprehensive than LAVI. For example, using maps of the deformation modes, we can visualize both regional and global shape variations, both of which may indicate potential for recurrence.

Unlike in [7], we decided to quantify anatomical variability using a registration approach based on diffeomorphism. This allows for finer shape variations to be captured without the need for pre-defined markers. In statistical analysis, we used partial least squares (PLS) regression rather than principal component analysis (PCA) since PLS regression recognizes the components that are directly linked to recurrence. We included a two-step regression to compare shape features and volume index. Finally, we used computed tomography (CT) to ensure detailed and accurate anatomical descriptions.

\section{Methods and Experiments}

\subsection{Data Preparation}

Population 40 paroxysmal AF (PAF) patients were studied. They had no previous ablation at the time of imaging. Average age was $59 \pm 11$ years old and $31 / 40$ of the patients are male. Post-ablation recurrence within six months was observed in 13/40 patients. Meanwhile, we chose 24 control subjects without significant difference in age or gender from the PAF group.

Image Acquisition All subjects were imaged before catheter ablation using a 64-slice Siemens SOMATOM CT scanner [8]. Multi-detector CT was performed, during the intravenous injection of iodinated contrast agent. The scans were ECG-gated for acquisition window to occur at end-systole, when LA motion is minimal. Temporal resolution was $66 \mathrm{~ms}$. The trans-axial images were acquired with a slice thickness of $0.5 \mathrm{~mm}$ and reconstructed with a voxel size of $0.5 \times$ $0.4 \times 0.4 \mathrm{~mm}^{3}$. The protocol of this study was approved by the local research ethics committee.

Endocardium Segmentation We segmented the LA endocardium from CT images using a region growing algorithm, as described in [9]. Semi-automatically drawn polygons isolated the left atrium and served as the boundary for succedent region 
growing process. A patient-specific intensity threshold, computed from tissue sampling, controlled the growing of blood pool during the iteration process until it touched the frontier of the endocardium. Pulmonary veins were cut several centimetres from the ostia. Based directly on image intensity, our segmentation can achieve high accuracy with such contrast CT, as well as identify PVs with several kinds of anatomies 3 .

Mesh Generation Next, we generated 3D volume tetrahedral meshes of the LA relaying on restricted Delaunay triangulation 11 11 . Range for size and angle of mesh elements were controlled via input parameters. To meet the requirements of implementation, we extracted surface triangular meshes of the LA from the volume meshes.

\subsection{Quantification of Shape Variations}

We encoded LA shape by deformation parameters in this step, adopting diffeomorphometry. The term was introduced recently and refers to the comparison of shapes and forms using a metric structure based on diffeomorphism. Methods under this framework have been proven to be efficient to qualify anatomical configurations and their differences for computational anatomy studies [12].

Mathematical currents, varifold metric, diffeomorphism First, the shape $S$ was represented by flux of 3D vector field/current $w$ across the surface, that belongs to a Gaussian reproducing kernel Hilbert space (RKHS) $W$ with kernel $K_{w}$. The current $w$ was parameterized by a set of $\vec{\delta}_{c_{k}}$, attached to distinct points on surface $S$.

Then, the deformation $\phi$ was estimated using the large deformation diffeomorphic metric mapping (LDDMM) on surface, characterized by initial velocity $\vec{v}_{0}$. The velocity vector belongs to a Gaussian RKHS $V$ with kernel $K_{V}$, and was weighted by a set of moment vectors $\vec{\alpha}_{c_{k}}$. Unless otherwise stated, $\left\{c_{k}\right\}_{k=1, \ldots, N_{c p}}$ refers to the control points of deformation in the following text. At point $x$, velocity

$$
v(x)=\sum_{k=1}^{N_{c p}} K_{V}\left(x, c_{k}\right) \vec{\alpha}_{c_{k}} .
$$

Thus, for patient $\# i$, the surface $S_{i}$ could be represented as the sum of deformed template $T$ and some residuals $\epsilon_{i}$, as

$$
S_{i}=\phi_{i} T+\epsilon_{i}
$$

For registration, we minimized the residuals $\epsilon$, in other words, the distance between $S_{i}$ and $\phi_{i} T$. For atlas construction, we minimized the sum of distances

\footnotetext{
${ }^{3}$ We used the MUSIC software for endocardium segmentation $\| 10$.

${ }^{4}$ We used CGAL 3D mesh generation algorithm http://http://doc.cgal.org/ latest/Mesh_3/index.html.
} 
from the template to every mesh, resulting in a Fréchet mean of shape complex,

$$
T=\underset{T \in W}{\arg \min } \sum_{i} d_{W}^{2}\left(T, S_{i}\right)
$$

The template and the deformations were estimated simultaneously.

In order to be independent of the surface orientation, the distance between surface meshes was defined based on varifold metric [13] as

$$
\begin{gathered}
d_{W}\left(S, S^{\prime}\right)^{2}=|| S-S^{\prime} \|_{W^{*}}^{2}=\langle S, S\rangle_{W^{*}}+\left\langle S^{\prime}, S^{\prime}\right\rangle_{W^{*}}+\left\langle S, S^{\prime}\right\rangle_{W^{*}} \\
\text { with }\left\langle S, S^{\prime}\right\rangle_{W^{*}}=\sum_{p} \sum_{q} K_{W}\left(c_{p}, c_{q}^{\prime}\right) \frac{\left(n_{p}^{T} n_{q}^{\prime}\right)^{2}}{\left|n_{p}^{T}\right|\left|n_{q}^{\prime}\right|}
\end{gathered}
$$

where $c$ (resp. $c^{\prime}$ ) refers to control points on surface $S$ (resp. $S^{\prime}$ ) and $n$ (resp. $n^{\prime}$ ) denotes normals related to controls points.

The line search strategy was used in optimization process. More details can be found in [14].

Remodeling A rigid alignment of all meshes was performed in the first place, to reduce the impact of different origin in $\mathrm{CT}$ images. Iterative closest point algorithm was applied to calculate an optimized rigid registration in the least square sense.

Using methods described above, we computed a 3D template of the LA shape in the control group. The template offered a reference for anatomical invariant. Then, we warped this template towards each mesh of PAF patients and calculated its deformation moments. We illustrate the process in Fig. 1 (a)

Deformations were parameterized as moment vectors $\vec{\alpha}_{c_{k}}$ attached to a same set of control points on the template for all patients. We use $\boldsymbol{M}_{i}$ to represent the deformation moments for patient $\# i$

$$
M_{i}=\left[\begin{array}{llllll}
\vec{\alpha}_{c_{1}}^{i} & \vec{\alpha}_{c_{2}}^{i} & \cdots & \vec{\alpha}_{c_{k}}^{i} & \cdots & \vec{\alpha}_{c_{N_{c p}}}^{i}
\end{array}\right]^{T}
$$

where $\vec{\alpha}_{c_{k}}^{i}=\left[\alpha_{c_{k} 1}^{i} \alpha_{c_{k} 2}^{i} \alpha_{c_{k} 3}^{i}\right]^{T}$ is the deformation moment vector associated with control point $\# k$ for patient \# $\# ; N_{c p}$ is the total number of control points.

Kernel width parameters were set as: varifold kernel width $\sigma_{W}=10 \mathrm{~mm}$ and deformation kernel width $\sigma_{V}=10 \mathrm{~mm}$, ensuring respectively a suitable scale of shape variations and deformation to capture. $N_{c p}=3952$ control points were placed near most variable parts on the template and helped in representing shape differences between the template and the meshes of PAF patients. With chosen parameters, atlas estimation and pairwise registration were performed efficiently with surfaces, as shown in Fig. 11(b).

\footnotetext{
${ }^{5}$ Atlas estimation and registration have been integrated in the Deformetrica software http://www.deformetrica.org/
} 


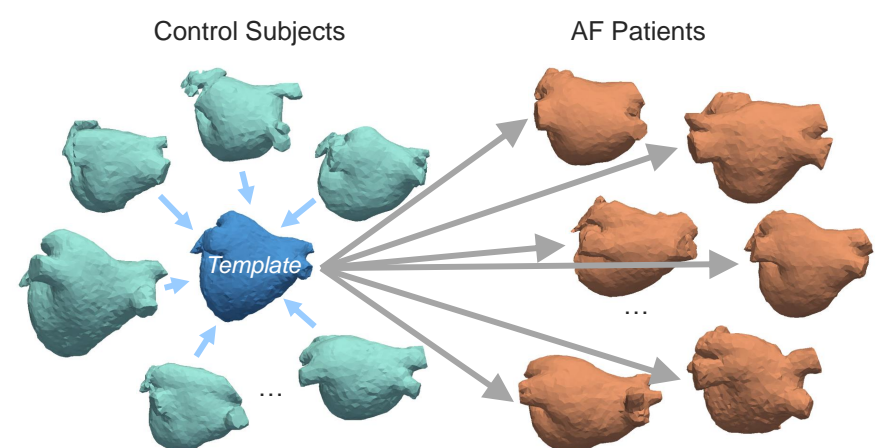

(a)

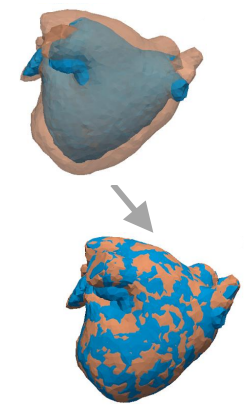

(b)

Fig. 1. Extraction of remodeling information vs. controls. (a) reference for atrial remodeling offered by control subjects; (b) the template in blue, before and after warping in a registration process toward the target in orange.

Whitening transform Then, we used whitening transform to reduce the correlation between the deformation moments.

According to Eq. 1, the norm of the velocity vector for patient \#i

$$
\left\|v^{i}\right\|^{2}=\left[\begin{array}{llll}
\vec{\alpha}_{c_{1}}^{i} & \vec{\alpha}_{c_{2}}^{i} & \cdots & \vec{\alpha}_{c_{N_{c p}}}^{i}
\end{array}\right] \boldsymbol{K}\left[\begin{array}{llll}
\vec{\alpha}_{c_{1}}^{i} & \vec{\alpha}_{c_{2}}^{i} & \cdots & \vec{\alpha}_{c_{N_{c p}}}^{i}
\end{array}\right]^{T}=\boldsymbol{M}_{i}^{T} \boldsymbol{K} \boldsymbol{M}_{i},
$$

where $\boldsymbol{K}$ is a positive definite matrix that defined a metric, with its blocks $K_{i j}=K_{V}\left(c_{i}, c_{j}\right) \otimes \mathbb{1}_{3}$. We used the Gaussian kernel $K_{V}(x, y)=\exp \left(\frac{-|x-y|^{2}}{\sigma_{V}^{2}}\right)$. Since the velocity vector $v$ belongs to a RKHS space, the whitened moment $\boldsymbol{K}^{1 / 2} \boldsymbol{M}_{i}$ belongs to the Euclidean space with $L_{2}$ norm. This allows us to apply seamlessly standard statistical methods like PLS.

We arranged the whitened deformation moments $\boldsymbol{K}^{1 / 2} \boldsymbol{M}_{i}$ into a matrix as

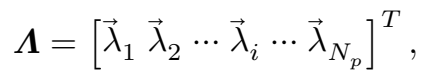

where $\vec{\lambda}_{i}$ is a column vector that contains all the elements in $\boldsymbol{K}^{1 / 2} \boldsymbol{M}_{i} ; N_{p}=$ 40 is the total number of patient. Thus, the dimension of $\boldsymbol{\Lambda}$ turns out to be $N_{p} \times\left(3 N_{c p}\right)$.

To sum up, modeling complex geometries with mathematical currents avoided using pre-defined markers, and therefore has the potential to summarize any shape feature related to certain clinical factors. The finite dimensional approximation of shape as deformation moments was robust to detect subtle anatomical differences. Finally, whitening transform reduced the correlation due to the kernel metric and constructed a $L_{2}$ space for statistical analysis. 


\subsection{Statistical Modeling}

PLS regression combines PCA with linear regression by projecting inputs $\boldsymbol{X}$ and $\boldsymbol{Y}$ to a new space. $\boldsymbol{X}$ is a $n \times m$ matrix of $m$ predictors for $n$ observations, and $\boldsymbol{Y}$ is a $n \times w$ matrix of $w$ response variables for $n$ observations. It relates predictors directly to response variables by finding multidimensional directions in $X$ that explain the maximum variance in $Y$. Here, we use same notations as the tutorial [15].

We chose PLS regression so as to find the principle dimensions in deformation parameters that correlate with post-ablation recurrence.

Dependency analysis with left atrial volume In the first PLS regression, the whitened deformation parameters were considered as predictors, while the LAVI was considered as response variable, as

$$
\boldsymbol{X}=\boldsymbol{\Lambda} \quad \text { and } \quad Y=\left[\begin{array}{lllllllll}
L A V I_{1} & L A V I_{2} & \cdots & L A V I_{i} & \cdots & L A V I_{N_{p}}
\end{array}\right]^{T},
$$

where $\boldsymbol{\Lambda}$ is defined in Eq. 7; $L A V I$ was calculated for each subject as $L A V I=$ $V_{L A} / B S A$, based on the size of the atrium $V_{L A}$ in units of $m L$ and the body surface area $B S A$ in units of $m^{2}$.

According to the percentage of variance explained shown in Table 1, the first mode spanned an optimal subspace that explained $84.61 \%$ of LAVI variance for the population under study. It was expected that this principle mode of variation would be linked to volume change. To analyze shape features which are complementary to the atrial size, we subtracted, for every patient, the components in deformation projected on this mode, as

$$
\boldsymbol{\Lambda}^{\prime}=\boldsymbol{X}-\boldsymbol{T}(:, 1) \boldsymbol{P}(:, 1)^{T}
$$

where $\boldsymbol{\Lambda}^{\prime}$ is the matrix of volume-reduced deformation parameters; $\boldsymbol{T}(:, 1)$ is the first column of X score matrix $\boldsymbol{T} ; \boldsymbol{P}(:, 1)$ is the first column of X loading matrix $\boldsymbol{P}$, referring to the first PLS component of deformation related to LAVI.

Table 1. Percentage of variance explained in the first partial least squares regression, for shape and left atrial volume index respectively.

\begin{tabular}{|c|c|c|c|c|c|}
\hline PLS components & $1^{\text {st }}$ & $2^{\text {nd }}$ & $3^{\text {rd }}$ & $4^{t h}$ & $5^{t h}$ \\
\hline Percentage of shape variance \% & 53.45 & 7.09 & 7.50 & 4.47 & 5.70 \\
\hline Percentage of LAVI variance \% & 83.61 & 5.45 & 2.03 & 2.07 & 0.96 \\
\hline
\end{tabular}

We therefore created a new matrix of deformation parameters that were not linearly related to LA volume change. 
Correlation with post-ablation recurrence In the second PLS regression, we studied the correlation between atrial shape and post-ablation recurrence, applying discriminant analysis and leave-one-out prediction. The volume-reduced deformation parameters were considered as predictors, while the post-ablation outcome within six months was considered as response variable.

For patient \# $i$, PLS regression was performed among all the other patients as

$$
\begin{aligned}
& \boldsymbol{X}=\boldsymbol{\Lambda}^{\prime}([1: i-1, i+1: \text { end }],:) \\
& Y=\left[\begin{array}{lll}
R_{1} R_{2} \cdots R_{i-1} R_{i+1} \cdots R_{N_{p}}
\end{array}\right]^{T},
\end{aligned}
$$

where $\boldsymbol{\Lambda}^{\prime}$ was computed based on Eq. 8; $R_{i}$ represents the post-ablation outcome within six months for patient $\# i$, with $R_{i}=1$ standing for with recurrence, $R_{i}=0$ without recurrence.

Then, we projected the deformation parameters of patient $\# i$ on the subspace constructed by the first $n$ PLS components, to calculate a predicted response for this new observation

$$
S I_{i}=\boldsymbol{\Lambda}^{\prime}(i,:) \boldsymbol{P}(:, 1: n) \boldsymbol{B}(1: n, 1: n) \boldsymbol{Q}(:, 1: n)^{T},
$$

where $\boldsymbol{\Lambda}^{\prime}(i,:)$ is the volume-reduced deformation parameters for patient \# $i ; \boldsymbol{P}$ refers to the X loading matrix; $\boldsymbol{B}$ is the diagonal matrix of coefficients $b_{h} ; \boldsymbol{Q}$ is the $\mathrm{Y}$ loading matrix.

We repeated the leave-one-out regression and prediction, and thus obtained shape indices for every patient $\left\{S I_{i}\right\}_{i=1,2, \ldots N_{p}}$ which qualified their potential for recurrence.

\section{Results}

We present here the first three modes of deformation, with a higher signal-tonoise ratio, and their prediction capabilities. Beyond the third mode, components may be dominated by noise, since the percentage of variance explained by them was smaller than half of the gap between the percentage of variance explained by previous two successive modes. The percentage of variance explained is shown in Table 2.

Table 2. Percentage of variance explained in the second partial least squares regression, for shape and post-ablation recurrence respectively.

\begin{tabular}{|c|c|c|c|c|c|}
\hline PLS components & $1^{\text {st }}$ & $2^{\text {nd }}$ & $3^{\text {rd }}$ & $4^{\text {th }}$ & $5^{\text {th }}$ \\
\hline Percentage of shape variance \% & 21.44 & 9.02 & 14.30 & 9.71 & 5.69 \\
\hline Percentage of recurrence variance \% & 27.47 & 18.09 & 5.67 & 3.98 & 3.95 \\
\hline
\end{tabular}

The principle modes of deformation related to post-ablation recurrence can be visualized and interpreted, shown in Fig. 2. Area strain [16] and the magnitude 
of displacement were mapped onto meshes to illustrate detailed variations in deformations. For area strain, red indicates enlargement of triangular elements, while blue indicates shrinking. For displacement, regions in red deform with a larger scale of displacement than regions in blue.

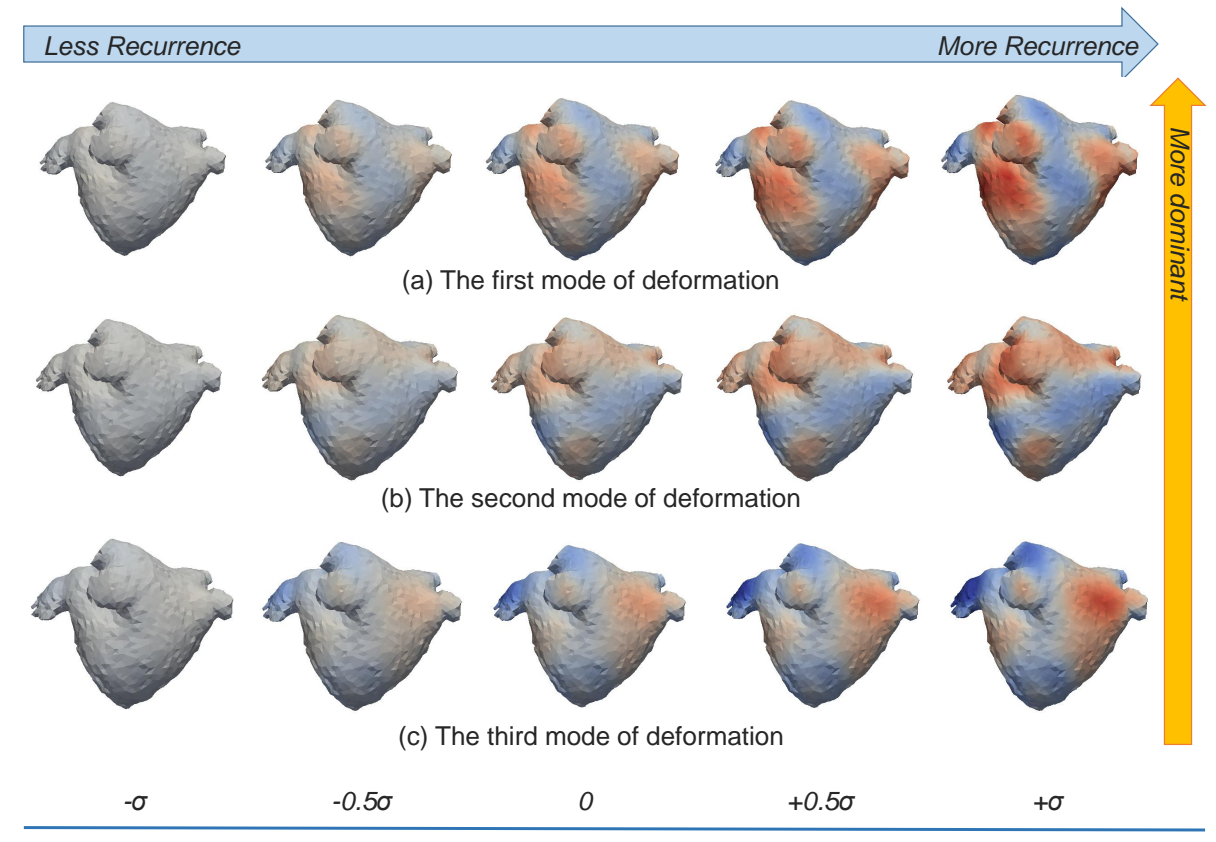

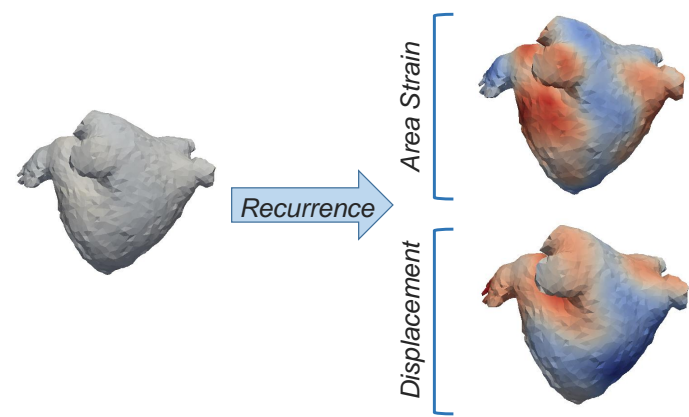

(a)

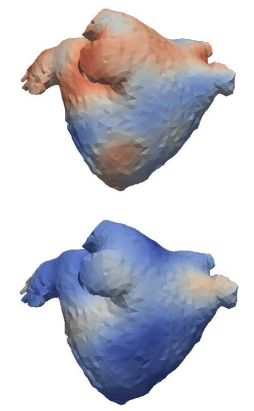

(b)

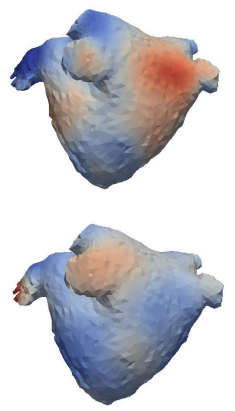

(c)

Fig. 2. The first three modes of deformation involved in post-ablation recurrence. $\sigma$ represents the standard derivation on each mode in the population.

Results show that the most dominant mode has an emphasis on regions underneath pulmonary veins, both with area strain and displacement of mesh elements. Clinical experts confirmed that this is one of the most complicated area to ablate in pulmonary vein isolation. Also, the remodelling around pulmonary 
veins seems to be an important aspect of AF. The second mode contains a twist of the upper-left part of the LA (from the posterior view, including roof, lateral and anterior segments) and also changes in orientation of pulmonary veins. The third mode, from less recurrence to more recurrence, reflects a slight change of roundness. These $3 \mathrm{D}$ deformation sequences can also be shown as videos in order to be more explicit. However, the subtle regional variations need further interpretation.

We compared prediction capabilities of shape indices with that of LAVI, using student's t-test and receiver operating characteristic (ROC) curves. The indices are shown in Table 3 .

Table 3. Student's t-test to compare shape indices and left atrial volume index in two sub-groups and area under receiver operating characteristic curves.

\begin{tabular}{|c|c|c|c|c|}
\hline PAF patients & Non-recurrence & Recurrence & P-value & AUC \\
\hline$S I$ - 1 PLS component & $0.28 \pm 0.20$ & $0.41 \pm 0.23$ & 0.04 & 0.65 \\
$S I$ - 2 PLS components & $0.29 \pm 0.22$ & $0.48 \pm 0.26$ & 0.01 & 0.73 \\
$S I$ - 3 PLS components & $0.30 \pm 0.21$ & $0.51 \pm 0.34$ & 0.01 & 0.70 \\
\hline Volume index $/ m L / m_{2}$ & $64.25 \pm 19.34$ & $64.97 \pm 22.23$ & 0.46 & 0.47 \\
\hline
\end{tabular}

- Shape indices derived from PLS regression differed significantly between recurrence and non-recurrence groups, with p-value reaching $p=0.01<0.05$ with the first two components, while LAVI did not, with $p=0.46$.

- Shape indices show a better classification performance as the discrimination threshold varies. We draw ROC curves of the shape indices and the volume index in Fig. 3. The area under the ROC curve (AUC) using the first two components is 0.73 , compared with 0.47 for LAVI.

- With discrimination threshold $t=$ 0.36 for shape index with the first two components, we predicted recurrence with 0.77 sensitivity and 0.67 specificity.

From the differences, we can conclude

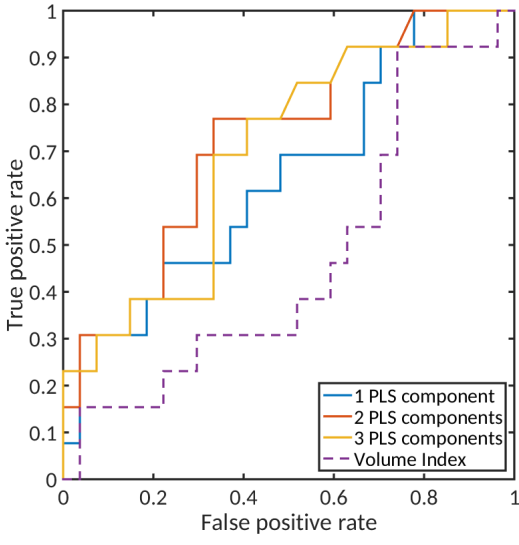

Fig. 3. Receiver operating characteristic curves of 6 -month post-ablation recurrence prediction. that the shape analysis discovered extra anatomical features compared with the volume index for the understanding of post-ablation recurrence. Meanwhile, the principle modes of deformation revealed in this study turned out to be clinically meaningful. 


\section{Conclusion}

We adapted a shape-based statistical model, extended from mathematical currents and diffeomorphism, to address the problem of post-ablation recurrence. We eliminated the impact of atrial size in shape analysis. Then, from regressions, we obtained shape indices better predicting post-ablation recurrence, when compared with dilatation. Our shape analysis approach summarizes all shape variations at a scale that is greater than the kernel width. It is also more robust, with respect to training samples, than using only one shape parameter, as is done with the volume index.

From a clinical viewpoint, we revealed the principal LA deformation modes that were related to a higher post-ablation recurrence rate. We visualized them and some anatomical details were consistent with the complexity of ablation in clinical practice. These features also bring new insights on how a shape evolves during LA remodeling, apart from volume change.

Future work includes using a larger database to reduce random effects even further, as well as combining shape variations with other factors, such as age, sex and electrocardiography, to stratify the risk of recurrence.

Acknowledgments. Part of the research was funded by the Agence Nationale de la Recherche (ANR)/ERA CoSysMed SysAFib and ANR MIGAT projects. The authors would like to thank Alan Garny, Côme Le Breton and Marco Lorenzi for their great support.

\section{References}

1. Massimo Zoni-Berisso, Fabrizio Lercari, Tiziana Carazza, Stefano Domenicucci, et al. Epidemiology of atrial fibrillation: European perspective. Clin Epidemiol, 6:213-220, 2014.

2. Antonio Berruezo, David Tamborero, Lluis Mont, Begoña Benito, Jose María Tolosana, Marta Sitges, Bárbara Vidal, Germán Arriagada, Francisco Méndez, Maria Matiello, et al. Pre-procedural predictors of atrial fibrillation recurrence after circumferential pulmonary vein ablation. European heart journal, 28(7):836$841,2007$.

3. Nikolaos Dagres, Hans Kottkamp, Christopher Piorkowski, Sebastian Weis, Arash Arya, Philipp Sommer, Kerstin Bode, Jin-Hong Gerds-Li, Dimitrios Th Kremastinos, and Gerhard Hindricks. Influence of the duration of holter monitoring on the detection of arrhythmia recurrences after catheter ablation of atrial fibrillation: implications for patient follow-up. International journal of cardiology, 139(3):305306, 2010.

4. Sung-Hee Shin, Mi-Young Park, Woong-Jin Oh, Soon-Jun Hong, Hui-Nam Pak, Woo-Hyuk Song, Do-Sun Lim, Young-Hoon Kim, and Wan-Joo Shim. Left atrial volume is a predictor of atrial fibrillation recurrence after catheter ablation. Journal of the American Society of Echocardiography, 21(6):697-702, 2008.

5. Felipe Bisbal, Esther Guiu, Naiara Calvo, David Marin, Antonio Berruezo, Elena Arbelo, José Ortiz-Pérez, Teresa María Caralt, José María Tolosana, Roger Borràs, 
et al. Left atrial sphericity: a new method to assess atrial remodeling. impact on the outcome of atrial fibrillation ablation. Journal of cardiovascular electrophysiology, 24(7):752-759, 2013.

6. Marrouche NF, Wilber D, Hindricks G, and et al. Association of atrial tissue fibrosis identified by delayed enhancement mri and atrial fibrillation catheter ablation: The decaaf study. JAMA, 311(5):498-506, 2014.

7. Marta Varela, Felipe Bisbal, Ernesto Zacur, Antonio Berruezo, Oleg Aslanidi, Lluis Mont, and Pablo Lamata. Novel computational analysis of left atrial anatomy improves prediction of atrial fibrillation recurrence after ablation. Frontiers in Physiology, 8:68, 2017.

8. S. Labarthe, Y. Coudiere, J. Henry, and H. Cochet. A semi-automatic method to construct atrial fibre structures: A tool for atrial simulations. In 2012 Computing in Cardiology, pages 881-884, Sept 2012.

9. Shuman Jia, Loïc Cadour, Hubert Cochet, and Maxime Sermesant. STACOMSLAWT Challenge: Left Atrial Wall Segmentation and Thickness Measurement Using Region Growing and Marker-Controlled Geodesic Active Contour. In 7th International Statistical Atlases and Computational Modeling of the Heart (STACOM) Workshop, Held in Conjunction with MICCAI 2016, volume 10124 of LNCS, pages 211-219. Springer, Mar 2017.

10. Hubert Cochet, Rémi Dubois, Frédéric Sacher, Nicolas Derval, Maxime Sermesant, Mélèze Hocini, Michel Montaudon, Michel Haïssaguerre, François Laurent, and Pierre Jaïs. Cardiac arrythmias: multimodal assessment integrating body surface ecg mapping into cardiac imaging. Radiology, 271(1):239-247, 2013.

11. Clément Jamin, Pierre Alliez, Mariette Yvinec, and Jean-Daniel Boissonnat. Cgalmesh: a generic framework for delaunay mesh generation. ACM Transactions on Mathematical Software (TOMS), 41(4):23, 2015.

12. Michael I Miller, Laurent Younes, and Alain Trouvé. Diffeomorphometry and geodesic positioning systems for human anatomy. Technology, 2(01):36-43, 2014.

13. Nicolas Charon and Alain Trouvé. The varifold representation of nonoriented shapes for diffeomorphic registration. SIAM Journal on Imaging Sciences, 6(4):2547-2580, 2013.

14. Stanley Durrleman, Marcel Prastawa, Nicolas Charon, Julie R Korenberg, Sarang Joshi, Guido Gerig, and Alain Trouvé. Morphometry of anatomical shape complexes with dense deformations and sparse parameters. NeuroImage, 101:35-49, 2014.

15. Paul Geladi and Bruce R Kowalski. Partial least-squares regression: a tutorial. Analytica chimica acta, 185:1-17, 1986.

16. Sebastiaan A Kleijn, Mohamed FA Aly, Caroline B Terwee, Albert C van Rossum, and Otto Kamp. Three-dimensional speckle tracking echocardiography for automatic assessment of global and regional left ventricular function based on area strain. Journal of the American Society of Echocardiography, 24(3):314-321, 2011. 